\title{
Superconducting pipes and levitating magnets
}

\author{
Yan Levin* and Felipe B. Rizzato ${ }^{\dagger}$ \\ Instituto de Física, Universidade Federal do Rio Grande do Sul Caixa Postal 15051, 91501-970, Porto Alegre, RS, Brazil
}

(Received 31 May 2006; published 12 December 2006)

\begin{abstract}
Motivated by a beautiful demonstration of the Faraday and the Lenz laws in which a small neodymium magnet falls slowly through a conducting nonferromagnetic tube, we consider the dynamics of a magnet falling coaxially through a superconducting pipe. Unlike the case of normal conducting pipes, in which the magnet quickly reaches the terminal velocity, inside a superconducting tube the magnet falls freely. On the other hand, to enter the pipe the magnet must overcome a large electromagnetic energy barrier. For sufficiently strong magnets, the barrier is so large that the magnet will not be able to penetrate it and will be levitated over the mouth of the pipe. We calculate the work that must done to force the magnet to enter a superconducting tube. The calculations show that superconducting pipes are very efficient at screening magnetic fields. For example, the magnetic field of a dipole at the center of a short pipe of radius $a$ and length $L \gtrsim a$ decays, in the axial direction, with a characteristic length $\xi \approx 0.26 a$. The efficient screening of the magnetic field might be useful for shielding highly sensitive superconducting quantum interference devices. Finally, the motion of the magnet through a superconducting pipe is compared and contrasted to the flow of ions through a trans-membrane channel.
\end{abstract}

DOI: 10.1103/PhysRevE.74.066605

PACS number(s): 41.20.Gz, 74.25.Ha, 07.55.Nk

\section{INTRODUCTION}

There is a beautiful demonstration of Faraday's and Lenz's laws which became very popular as a result of an easy availability of powerful rare earth magnets [1-9]. The demonstration consists of a long pipe made of a conducting, nonferromagnetic material, such as copper or aluminum, and a neodymium magnet which is allowed to fall through it. One finds that the magnet takes a very long time to traverse the pipe. In fact for a tube of about $2 \mathrm{~m}$ in length, the magnet takes almost $25 \mathrm{~s}$ to finish the trip [9]. On the other hand, a nonmagnetic object of the same dimensions falls through the pipe in less than $1 \mathrm{~s}$. It is quite amazing to observe the falling magnet from the top aperture, the magnet appears to be moving through a very dense fluid. In reality, air provides only a negligible resistance, and what actually slows the magnet is the force produced by the eddy currents induced in the pipe. This force is proportional to the velocity of the falling magnet. When the drag force becomes equal to the magnet's weight, acceleration ceases and the fall continues at a constant terminal velocity. For strong rare earth magnets, the terminal velocity is reached very quickly. Perhaps surprisingly, in view of the complexity of the problem, it is actually possible to perform a fairly simple calculation which agrees quantitatively with the terminal velocity observed experimentally [9]. Curiously, the calculation also predicts that the terminal velocity should be proportional to the electrical resistivity of the pipe's material. This suggests that if the pipe is an ideal superconductor, the velocity of the falling magnet should vanish. One can easily see, however, that this conclusion cannot be right. Suppose that a magnetic dipole is created inside an infinite superconducting pipe. During the process of creation, the magnetic field inside the pipe is

\footnotetext{
*Electronic address: levin@if.ufrgs.br

${ }^{\dagger}$ Electronic address: rizzato@if.ufrgs.br
}

changing and the electric current is induced on its surface. The surface currents screen the magnet's field and prevent it from entering into the interior of the superconductor. In the case of an ideal superconductor (inertialess electrons) considered in the bulk of this paper, the penetration length is zero and both the magnetic and the electric fields are perfectly screened. By symmetry it is also clear that for an infinitely long pipe, the magnetic field produced by the induced currents is maximum precisely at the location of the magnet. Since the magnetic force on a dipole is proportional to the gradient of the field it must, therefore, vanish so that the magnet will fall without any resistance. The theory of Ref. [9] is applicable neither to superconductors nor to very good conductors of vanishing resistivity, since it was explicitly constructed to treat normal metals for which the magnetic permeability is very close to that of vacuum. Furthermore, the rate of decay of the induced currents in such metals is very fast, compared to the magnet fall velocity, allowing us to neglect the effects of self-induction [9]. Clearly, neither one of these conditions is met in the case of super or ideal conductors which dynamically screen magnetic field from their interior. As the resistivity of the pipe metal is decreased, there will be a crossover from the terminal velocity found in Ref. [9] for normal pipes to the free fall velocity inside perfectly conducting pipes.

Although a magnet "created" in the interior of an infinite superconducting pipe will fall freely under the action of the gravitational field, it takes work to bring (create) the magnet inside the pipe in the first place. This is so because the magnetic field lines, which for a free dipole spread throughout the space, must now be confined in a restricted volume. In this paper we will calculate the work that must be done to bring a magnet into a superconducting pipe of length $L$ and radius $a$. Furthermore, the formalism developed here can be easily extended to study more general problems of screening of the magnetic field in cylindrical geometry [3,4], which are of particular interest for the development of reliable superconducting quantum interference devices [5]. 


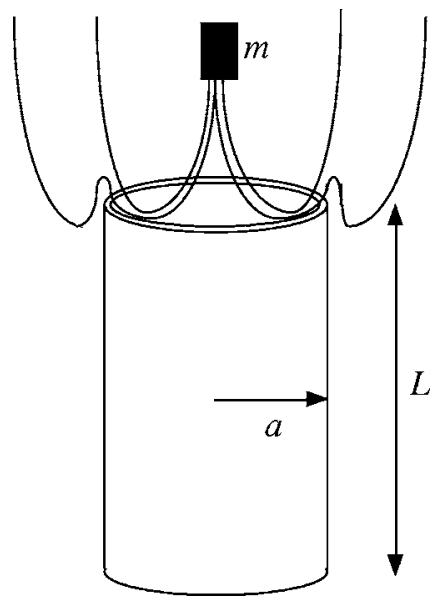

FIG. 1. Schematics representation of a magnetic dipole descending into a superconducting tube. The flux lines are repelled from the tube.

\section{THE MODEL}

The model that we shall study is depicted in Fig. 1. A magnetic dipole, of moment $\mathbf{m}=m \hat{\mathbf{z}}$, is brought from infinity and is inserted into cylindrical superconducting pipe of length $L$ and radius $a$ along the symmetry axis. This axis is taken to coincide with the $z$ axis of the coordinate system. The wall thickness of the pipe is assumed to be much smaller than the inner radius, and will therefore be ignored. The Faraday law of induction requires that

$$
\frac{d \Phi(z, t)}{d t}=-\oint \mathbf{E} \cdot d \mathbf{r},
$$

where $\Phi(z, t)$ is the magnetic flux passing through a cross section of the pipe at position $z$ and time $t$, and $\mathbf{E}$ is the local electric field. Since the tangential component of the electric field is continuous across the superconductor/air interface, the right-hand side of Eq. (1) must vanish because no electric field can be present inside a perfect conductor. Thus, the flux passing through any cross section of the pipe must be constant in time. Furthermore, when the magnet is at infinity, the flux entering the pipe is zero, which then means that $\Phi(z, t)=0$ at all future times as well. Vanishing flux is a direct consequence of the physics of cylindrical superconductors and must be used as a boundary condition for the solution of the Maxwell's equations. In Fig. 1 we have made an attempt to represent the magnetic field lines which are not allowed to go through the pipe because of the restriction on flux. Again we stress that this behavior is very different from what happens with normal metals [9] for which the electric field does not vanish and the magnetic flux changes through different cross sections of the pipe.

Inside the superconductor, magnetic field is zero and the continuity of the normal component of $\mathbf{B}$ requires that it also vanish at the superconducting wall. The magnetic field lines must, therefore, be tangent to the pipe's surface. In general, however, vanishing of $\mathbf{B}_{n}$ at the interface is not sufficient to fully specify the boundary condition necessary for the existence of a unique solution to Maxwell equations.

\section{UNIQUENESS}

What are the boundary conditions which will make the field equation

$$
\boldsymbol{\nabla} \times \mathbf{B}=\mu_{0} \mathbf{J}
$$

have a unique solution? Suppose that Eq. (2) allows for two distinct solutions $\mathbf{B}_{1}$ and $\mathbf{B}_{2}$ for the same value of the current density $\mathbf{J}$. Since $\boldsymbol{\nabla} \cdot \mathbf{B}=0$, we can always define a vector potential $\mathbf{A}$ such that $\mathbf{B}_{1,2}=\boldsymbol{\nabla} \times \mathbf{A}_{1,2}$. Now, consider the integral over all space

$$
\int \delta \mathbf{B} \cdot \delta \mathbf{B} d^{3} r=\int \boldsymbol{\nabla} \times \delta \mathbf{A} \cdot \boldsymbol{\nabla} \times \delta \mathbf{A} d^{3} r,
$$

where $\delta \mathbf{B} \equiv \mathbf{B}_{2}-\mathbf{B}_{1}$ and $\delta \mathbf{A} \equiv \mathbf{A}_{2}-\mathbf{A}_{1}$. The integrand on the right-hand-side can be cast into more convenient form using the identity $(\boldsymbol{\nabla} \times \delta \mathbf{A})^{2} \equiv \delta \mathbf{A} \cdot \boldsymbol{\nabla} \times \boldsymbol{\nabla} \times \delta \mathbf{A}+\boldsymbol{\nabla} \cdot(\delta \mathbf{A} \times \boldsymbol{\nabla}$ $\times \delta \mathbf{A})$. Recalling that the current sources for the fields 1 and 2 are identical: $0=\mu_{0}\left(\mathbf{J}_{2}-\mathbf{J}_{1}\right) \equiv \mu_{0} \delta \mathbf{J}=\boldsymbol{\nabla} \times \delta \mathbf{B}=\boldsymbol{\nabla} \times \boldsymbol{\nabla} \times \delta \mathbf{A}$, the integrand of Eq. (3) reduces to a perfect divergence. Using the divergence theorem, the volume integral can now be transformed into an integral over the bounding surfaces $S$, which in our case are the cylinder and the spherical shell of radius $R=\infty$ :

$$
\int \delta B^{2} d^{3} r=\int_{S} d a \hat{\mathbf{n}} \cdot(\delta \mathbf{A} \times \boldsymbol{\nabla} \times \delta \mathbf{A}) .
$$

Since the dipolar field decreases rapidly with distance, the contribution to the integral in Eq. (4) coming from the spherical shell at $R=\infty$ vanishes, and $S$ reduces to the surface of the cylinder. Using the invariance of the integrand under cyclical permutation of vectors, we see that Eq. (2) has a unique solution $[\delta \mathbf{B}(\mathbf{r}) \equiv 0]$ either if $\hat{\mathbf{n}} \times \delta \mathbf{A}=0$ or $\hat{\mathbf{n}} \times \delta \mathbf{B}$ $=0$, on the pipe surface. To have a well posed problem it is, therefore, not sufficient to specify only the normal component of the magnetic field, instead the tangential components of the field at the interface must be provided. In a cylindrical geometry the boundary conditions posed in terms of the vector potential are particularly useful. Azimuthal symmetry around the $z$ axis, restricts the vector potential to have only one non zero component in the $\mathbf{e}_{\phi}$ direction, $\mathbf{A}=A_{\phi} \hat{\mathbf{e}}_{\phi}(r, z)$. The flux through a cross section of the pipe is then

$$
\begin{aligned}
\Phi(z) & =\int \mathbf{B} \cdot \hat{\mathbf{n}} d a \\
& =\int \boldsymbol{\nabla} \times \mathbf{A} \cdot \hat{\mathbf{n}} d a=\oint \mathbf{A} \cdot d \mathbf{r}=2 \pi a A_{\phi}(a, z) .
\end{aligned}
$$

Specification of the flux passing through the pipe is, therefore, equivalent to the specification of $A_{\phi}$ and guarantees that Eq. (2) has a unique solution in the cylindrical geometry.

\section{A PIPE OF INFINITE LENGTH}

For superconducting pipes of $L=\infty$, Eq. (2) can be solved analytically, while for finite $L$ only numerical solution is possible. We start, therefore, with the $L=\infty$ case. 
The dipole of moment $\mathbf{m}=m \hat{\mathbf{z}}$ is located inside the pipe on the axis of symmetry at $z=0, r=0$. In the pipe's interior there are no free currents and the Ampere law (2), reduces to $\boldsymbol{\nabla}$ $\times \mathbf{B}=0$ outside the magnet. The magnetic field can then be written as a gradient of a scalar function $\mathbf{B}=-\nabla \varphi$. This defines the scalar magnetic potential $\varphi$, which also satisfies the Laplace equation since $\boldsymbol{\nabla} \cdot \mathbf{B}=0$. The magnetic potential $\varphi$ $\equiv \varphi_{d}+\varphi_{\text {ind }}$ is produced by the point dipole

$$
\varphi_{d}=\frac{\mu_{0} m z}{4 \pi\left(r^{2}+z^{2}\right)^{3 / 2}},
$$

and by the currents induced on the surface of the superconductor. The dipole potential can be written as a Fourier integral

$$
\varphi_{d}(r, z)=\frac{\mu_{0} m}{2 \pi^{2}} \int_{0}^{\infty} k K_{0}(k r) \sin (k z) d k .
$$

where $K_{0}$ is a modified Bessel function of the second kind. Since Eq. (6) is a solution of the Laplace equation, so must be $\varphi_{\text {ind }}$,

$$
\frac{1}{r} \frac{\partial}{\partial r} r \frac{\partial}{\partial r} \varphi_{\text {ind }}+\partial_{z}^{2} \varphi_{\text {ind }}=0,
$$

for $r<a$. We next note that $\varphi_{\text {ind }}$ must be odd in $z$, free of singularities, and vanish as $z \rightarrow \infty$. Under these conditions, the solution of the Laplace equation (8) can be written in terms of a Fourier integral involving a modified Bessel function of the first kind

$$
\varphi_{\text {ind }}(r, z)=\frac{2}{\pi} \int_{0}^{\infty} A(k) I_{0}(k r) \sin (k z) d k .
$$

According to the results of the previous section, the function $A(k)$ will be uniquely determined by the condition that $\Phi(z)=0$, which means that no magnetic field lines are lost to the wall. For infinite superconducting pipe this boundary condition, is, therefore, equivalent to the vanishing of the normal component of the magnetic field $B_{n}(a, z)=0$ on the superconductor surface. Thus the normal component of the induced field must cancel exactly the field produced by the dipole

$$
\partial_{r} \varphi_{\text {ind }}\left|r=a=-\partial_{r} \varphi_{d}\right|_{r=a}=\frac{\mu_{0} m}{2 \pi^{2}} \int_{0}^{\infty} k^{2} K_{1}(k a) \sin (k z) d k,
$$

at the air/superconductor interface. To arrive at the last equality of Eq. (10) we have used the identity $d K_{0}(x) / d x=$ $-K_{1}(x)$. Combining expressions (9) and (10) and using $d I_{0}(x) / d x=I_{1}(x)$, enables us to calculate the function

$$
A(k)=\frac{\mu_{0} m}{4 \pi} \frac{k K_{1}(k a)}{I_{1}(k a)} .
$$

The magnetostatic scalar potential inside an infinite superconducting pipe is then

$$
\varphi(r, z)=\frac{\mu_{0} m z}{4 \pi\left(r^{2}+z^{2}\right)^{3 / 2}}+\frac{\mu_{0} m}{2 \pi^{2}} \int_{0}^{\infty} d k k \sin (k z) \frac{K_{1}(k a)}{I_{1}(k a)} I_{0}(k r) .
$$

The first term on the right-hand side of Eq. (12) is the potential produced by the point dipole located at $r=0, z=0$, while the second term is the magnetostatic scalar potential produced by the electric currents induced on the superconducting surface. For large $z$, Eq. (12) simplifies to

$$
\varphi(r, z) \approx \frac{\mu_{0} m|z|}{2 \pi a^{2} z}\left(1+\frac{J_{0}\left(x_{1} r / a\right)}{J_{0}\left(x_{1}\right)^{2}} e^{-x_{1}|z| a}\right),
$$

where $x_{1}$ is the first root of the Bessel function $J_{1}: J_{1}\left(x_{1}\right)$ $=0$ with $x_{1} \approx 3.831$. One can arrive at Eq. (13) either by extending the integration in Eq. (12) to the complex plane, noticing that the poles of the integrand lie on the imaginary axis and are the roots of the Bessel function $J_{1}(x)$. Alternatively-and perhaps simpler-one can calculate the Green's function directly as an expansion in Bessel functions $J_{n}$.

The axial magnetic field of a dipole inside a superconducting pipe is

$$
B_{z}(r, z) \approx \frac{\mu_{0} m}{2 \pi a^{2}} \frac{k_{1} J_{0}\left(x_{1} r / a\right)}{J_{0}\left(x_{1}\right)^{2}} e^{-x_{1}|z| / a} .
$$

The field is exponentially screened, with a characteristic length $\xi=a / x_{1} \approx 0.26 a$.

To confine the magnet inside a superconducting tube of small radius costs a lot of energy. The work necessary to achieve this can be calculated using a charging process similar to the one employed in electrostatics- the magnetic moment is built up gradually, while the magnet interacts with the induced field of the superconductor. Since the induced field is linearly proportional to the magnetic strength, the charging process reduces to

$$
W=-\int_{0}^{1} d \lambda \mathbf{m} \cdot \mathbf{B}_{\text {ind }}(\lambda \mathbf{m})=-\frac{1}{2} \mathbf{m} \cdot \mathbf{B}_{\text {ind }}(\mathbf{m}) .
$$

The field $\mathbf{B}_{\text {ind }}$ can be easily obtained from the second term of Eq. (12), yielding

$$
W=\frac{\mu_{0} m^{2}}{4 \pi^{2} a^{3}} \int_{0}^{\infty} d x x^{2} \frac{K_{1}(x)}{I_{1}(x)} \approx 0.797 \frac{\mu_{0} m^{2}}{4 \pi a^{3}} .
$$

The condition that the magnetic field lines must exit the pipe on the same side on which they entered $[\Phi(z)=0]$, implies that the magnet can not "probe" the full length of the pipe. Thus, if the pipe length is such that $L \gtrsim a$, its field screening properties should be similar to those of a pipe of $L=\infty$. With this observation in mind we are now ready to study the superconducting pipes of finite length.

\section{PIPES OF FINITE LENGTH}

Consider a superconducting pipe of finite length $L$ placed along the $z$ axis, whose center coincides with the origin at $z=0$. Results of the previous sections suggests that if $L>a$, 
shortly after the dipole finds itself inside the tube, the magnetic field configuration should be identical to that inside an infinite pipe and the axial force should vanish. However, little can be said about what kind of forces act upon the dipole as it enters or exits the pipe. The finite length case should, therefore, be examined with some care.

From Secs. II and III we recall that the magnetic flux at any cross section of a superconducting pipe must vanish. This information will be central to the practical aspects of the theory from now on. As the dipole approaches the pipe from $z \rightarrow+\infty$, surface currents are generated over the pipe's surface. It is convenient to imagine that the length of the pipe is subdivided into uniform rings, each carrying a circulating surface current density $j(z)$. The vector potential produced by these currents has only $\phi$ component and its magnitude is given by the linear superposition

$$
A_{\text {ind }}(r, z)=\frac{a \mu_{0}}{2} \int_{-L / 2}^{+L / 2} K\left(r, z, z^{\prime}\right) j\left(z^{\prime}\right) d z^{\prime},
$$

where the kernel

$$
K\left(r, z, z^{\prime}\right)=\int_{0}^{\infty} d k e^{-k\left|z-z^{\prime}\right|} J_{1}(k r) J_{1}(k a)
$$

is obtained from the field produced by one thin ring [2]. The flux generated by the surface currents at $z$ is $\Phi_{\text {ind }}(z)$ $=2 \pi a A_{\text {ind }}(a, z)$, see Eq. (5). For $r=a$ the kernel can be evaluated explicitly in terms of the hypergeometric function

$$
K\left(a, z, z^{\prime}\right)=\frac{a_{2}^{2} F_{1}\left[3 / 2,3 / 2 ; 3 ;-\frac{4 a^{2}}{\left|z-z^{\prime}\right|^{2}}\right]}{2\left|z-z^{\prime}\right|^{3}} .
$$

The condition that through each cross section of the pipe the net flux vanishes $\Phi(z, t)=0$ leads to an integral equation for the surface currents

$$
\int_{-L / 2}^{+L / 2} K\left(z, z^{\prime}\right) j\left(z^{\prime}\right) d z^{\prime}=-\frac{m}{2 \pi\left[\left(z_{m}-z\right)^{2}+a^{2}\right]^{3 / 2}},
$$

where $z_{m}$ is the coordinate of the dipole. To determine $j(z)$, Eq. (20) is solved numerically by first discretizing the integral and then performing a matrix inversion. Once the current distribution is known, the vector potential $A_{\text {ind }}$ is calculated using Eq. (17). Knowing $A_{\text {ind }}$, the induced magnetic field on the axis of symmetry $r=0, \mathbf{B}_{\text {ind }}=\hat{\mathbf{z}} /\left.r \partial_{r}\left(r A_{\text {ind }}\right)\right|_{r=0}$ and the magnetic force on the dipole $\mathbf{F}=\left.\hat{\mathbf{z}} \partial_{z}\left(\mathbf{m} \cdot \mathbf{B}_{\text {ind }}\right)\right|_{z=z_{m}, r=0}$ can be easily evaluated. We note that forces arising from flux trapping in type-II superconductors are neglected in the present discussion. For this to be a good approximation, the superconductor must have either high critical state current density $J_{c}$ or be subjected to only small magnetic field [6-8]. We will discuss this further in conclusions.

\section{A. Checking the accuracy of the numerical procedure}

The accuracy of the numerical procedure used to solve the integral equation Eq. (20) can be judged by comparing it with the analytical solution for an infinite pipe, Eq. (12). In

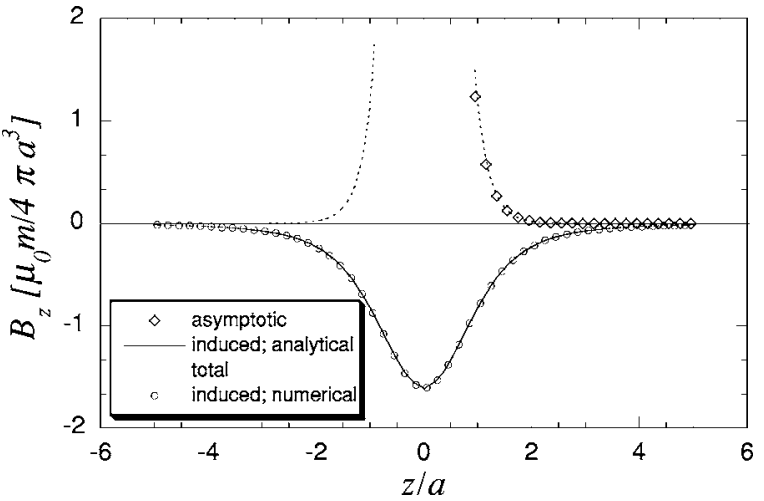

FIG. 2. Comparison of the numerical (circles) and the analytical (full line) calculations for the induced magnetic field. The analytical result is for $L=\infty$, while the numerical solution is for $L=10 \mathrm{a}$. Nevertheless, there is a perfect agreement between the two. We also compare the analytical asymptotic form (dotted line) Eq. (14) for the axial magnetic field, with the result of the numerical integration (squares) for $L=10 a$, showing a clear exponential decay of the axial field, even inside a finite superconducting pipe.

Fig. 2 we compare $B_{\text {ind }}$ along the axis of symmetry for an infinitely long pipe with the field calculated using a numerical integration of Eq. (20) for a pipe of $L=10 a$, located between $z=-5 a$ and $z=5 a$, with a point dipole at $z=z_{m}=0$. The agreement is perfect and attests to the reliability of the numerical solution. Furthermore, since the axial magnetic field is maximum at the position of the dipole, the force on it will vanish, in agreement with our previous discussion.

In Fig. 2 we also show that asymptotically the magnetic field is well approximated by Eq. (14). Thus, in agreement with the previous discussion, the axial magnetic field is screened exponentially even for pipes of finite length, as long as $L \gtrsim a$.

\section{B. End effects for superconducting tubes of finite lengths}

Satisfied with the accuracy of the numerical procedure, we can now use it to study the end effects associated with the finite length superconducting pipes. In Fig. 3 we plot the magnetic force felt by a magnet as it moves from infinity into the interior of a superconducting pipe of length $L=10 a$. Panel (a) shows a strong repulsive force near the pipe entrance which vanishes rapidly as the magnet penetrates into the pipe. In the case of small neodymium magnets (weight $6 \mathrm{~g}$ ) used in our demonstrations of the Faraday and the Lenz laws [9] and a superconducting pipe of radius $a=7.85 \mathrm{~mm}$ we find the repulsive force to be sufficient to support a weight of $1 \mathrm{~kg}$. In panel (b) we plot the work necessary to bring the magnet from infinity to a point $z$. Clearly, there is a large electromagnetic energy barrier that the magnet must overcome to enter the pipe. Note that soon after the magnet crosses the front edge into the interior of the tube, the potential stabilizes at a plateau the value of which is in perfect agreement with the work found to be necessary to confine a dipole inside an infinite superconducting tube, Eq. (16). This agreement is, again, a consequence of a very efficient exponential screening of the magnetic field by the surface cur- 

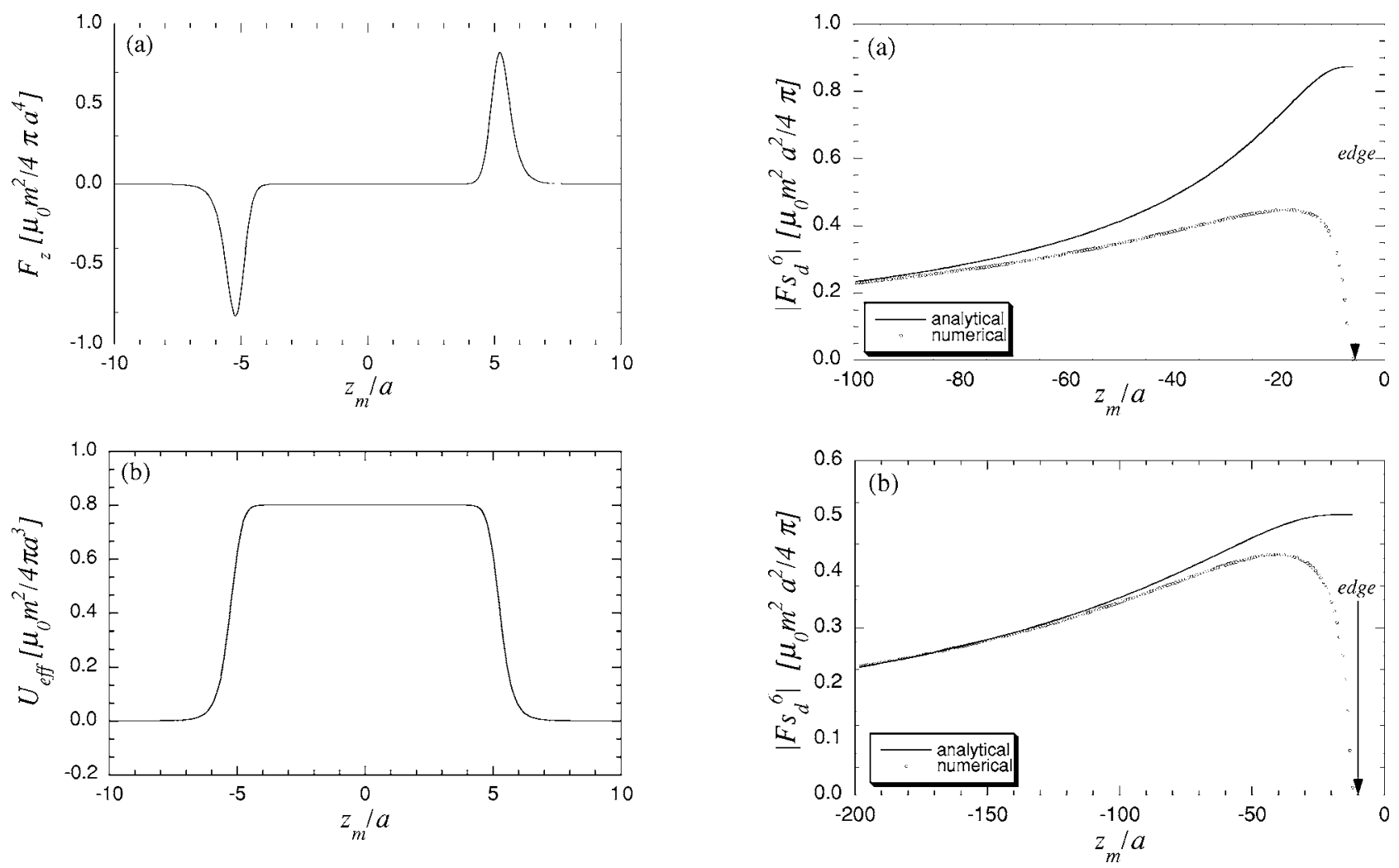

FIG. 3. Force (a) and the potential energy (b), for a dipole moving along the axis of symmetry of a superconducting pipe located between $[-5,5]$. Although each peak of the force looks rather symmetric, in reality the force decays as a power outside the pipe and exponentially inside.

rents, even inside pipes of finite length.

As the magnet approaches a superconducting tube, it experiences a magnetic force which opposes its motion. The magnitude of this force can be estimated using a simple scaling argument. Magnetic field produced by a dipole decays with distance as $B_{\text {magnet }} \sim m / s^{3}$. If the superconducting pipe is sufficiently narrow $(L \gg a)$ and the magnet is not too close to the front edge, the mutual-inductance effects between the sections of the pipe can be neglected and the induced surface current density at distance $s$ from the magnet will be $j(s)$ $\sim B_{\text {magnet }} \sim m / s^{3}$. On the other hand, each one of these current loops will produce a magnetic field at the position of the dipole, $d B_{\text {pipe } \rightarrow \text { magnet }} \sim j(s) d s / s^{3} \sim m d s / s^{6}$, and will result in a net force

$$
\left|F_{z}\right|=\alpha m^{2} \int_{s_{d}}^{s_{d}+L} d s / s^{7}=\frac{\alpha m^{2}}{s_{d}^{6}} f\left(\frac{s_{d}}{L}\right),
$$

where $s_{d}=\left|z_{m}\right|-L / 2$ is the distance of the magnet to the front edge of the pipe and $\alpha$ is the pipe's polarizability factor. The scaling function is found to be $f(x) \equiv 1-x^{6} /(1+x)^{6}$. Asymptotically, the force $F_{z}$ behaves as

$$
\left|F_{z} s_{d}^{6}\right| \rightarrow \begin{cases}\alpha m^{2} & \text { if } a \ll\left|s_{d}\right| \ll L, \\ 6 \alpha m^{2} L /\left|z_{m}\right| & \text { if }\left|s_{d}\right| \gg L .\end{cases}
$$

Thus, if $L \gg a$, the force on the magnet decays algebraically outside the pipe and vanishes exponentially after the magnet enters the pipe.

FIG. 4. Asymptotic force felt by a dipole when it is far from a superconducting pipe. Points are the result of the numerical calculation and the solid lines are the estimates obtained using Eq. (21). Panel (a) is for $L=10 a$ and panel (b) is for $L=20 a$.

The polarizability $\alpha$ can be calculated by considering the far asymptotic limit of Eq. (22). Under this condition, the magnetic field varies only slightly over the length of the pipe, and the induced current can be calculated analytically by solving Eq. (20). We find,

$$
I=\frac{m L}{2 \pi\left|z_{m}\right|^{3}} .
$$

This current will produce a magnetic field at the position of the dipole resulting in a repulsive force

$$
F=\frac{3 \mu_{0} m^{2} a^{2} L}{4 \pi\left|z_{m}\right|^{7}} .
$$

Comparing Eqs. (24) and (22) we find the polarizability of the superconducting pipe to be $\alpha=\mu_{0} a^{2} / 8 \pi$.

In Fig. 4(a) we compare the asymptotic force calculated using the numerical solution of the integral Eq. (20) with the estimate obtained using Eq. (21) for a tube of $L=10 a$. The agreement is very good for $\left|z_{m}\right| \gg L$, but poorer for smaller values of the coordinate. The problem is that $L=10 a$ is not sufficiently large to well satisfy the inequality $a \ll\left|z_{m}\right| \ll L$, as demanded by the first asymptotic region of Eq. (22). For larger values of $L$, such as $L=20 a$ of Fig. 4(b), the agreement already is much better. The abrupt decay of $F s_{d}^{6}$ near the 
entrance of the tube is a consequence of the finite cross section of the pipe, which becomes important for small values of $s_{d}$.

\section{CONCLUSIONS}

We have examined the forces and the fields produced by a small magnet as it enters into a superconducting pipe. For an infinitely long pipe, the magnetic field produced by the magnet inside the tube is exponentially screened by the surface currents.

In the case of finite superconducting pipes we find that the magnetic field is also exponentially screened, as long as the length of the pipe is larger than its diameter. The exponential screening of the magnetic field is a consequence of the Faraday law which leads to vanishing of the magnetic flux inside a superconducting tube. The flux lines can not go through the pipe and at any cross section the number of lines going down is the same as the number of lines coming up. The electromagnetic cost of confining a dipole inside a superconducting tube is, therefore, very large, since the field lines must be strongly compressed to fit inside the tube. We calculate that in order to insert a small neodymium magnet of $6 \mathrm{~g}$ into a superconducting pipe of radius $8 \mathrm{~mm}$ requires $10 \mathrm{~N}$ of force. Once the magnet is inside the pipe, however, its motion will continue unhindered. At the exit, the magnet will be ejected with the same force that was required to insert it into the pipe in the first place.

At this point it is interesting to reconsider the "falling magnet paradox." The calculations of Ref. [9] show that as the resistivity of the pipe's metal vanishes, the terminal velocity of the magnet approaches zero. This suggests that the magnet will become suspended inside the pipe. Clearly this contradicts the results derived in the present paper showing that the magnet will fall freely, after entering one tube radius into the superconducting pipe. How can these two seemingly diametrically opposite conclusions be reconciled? The answer to this question lies in the phenomenon of self induction. While the self induction could be safely ignored in the case of normal metals and sufficiently strong magnets considered in the Ref. [9], it can no longer be neglected in the limit of vanishing resistivity $\rho$. Specifically, it is possible to argue that the self induction can be ignored only when the fall velocity of the magnet is much less than the critical value $v_{c}=2 \rho / \mu_{0} d$, where $d$ is the thickness of the pipe [1]. Now consider a pipe made of a very good conductor (it does not have to be a superconductor) inside which a dipole is created at $t=0$. The creation process will induce the currents on the walls of the conductor which will screen the magnetic field of the dipole. However, if the conductivity of the metal is not identically zero, the field of the dipole will eventually leak out of the pipe. Before this happens, however, the magnet will fall under the action of the gravitational field. In the limit $\rho \rightarrow 0$ the velocity of the magnet will quickly reach $v_{c}$, and the self-induction will become relevant. Thus, for very good conductors the results of Ref. [9] will no longer apply. We conclude that there must be a crossover between the regime of normal metals, for which magnets reach terminal velocity, and the ideal conductors in which the magnets fall freely.

It is interesting to compare the magnet's motion through a superconducting pipe, to the flow of ions through an ion channel [11]. Ion channels are water filled holes, responsible for a small electric field that exists across all biological membranes. Since the dielectric constant inside a pore is much larger than the dielectric constant of a phospholipid membrane, the normal component of the electric field at the channel/membrane interface is very small and the electric field lines are mostly confined to stay in the pore's interior. In this respect, the pore is very similar to a superconducting pipe, for which the normal component of the magnetic field also vanishes on the boundary. Nevertheless, one finds that contrary to what happens to a magnet inside a superconductor, the repulsive force on an ion does not vanish even when it is far inside the channel. Ions always feel a force which tries to expel them from the channel (except exactly at the midpoint where, by symmetry, the force vanishes).

The difference between the superconducting pipes and the ion channels is precisely due to the additional constraint of vanishing flux imposed by the Faraday law on a perfectly conducing pipe. The requirement that the flux through any cross section of a superconducting pipe must vanish, forces the system into a metastable state in which the coaxial motion is free of any electromagnetic forces.

In this work we have neglected the hysterectic (flux trapping) forces typically present in type-II superconductors. For small cylindrical neodymium magnets weighing $6 \mathrm{~g}$ and radius $r=6.35 \mathrm{~mm}$ moving through a superconducting pipe of $a \sim 8 \mathrm{~mm}$, the magnetic field at the pipe surface is on the order of $B \sim 0.1 \mathrm{~T}$. Considering a strong pinning condition with a high critical state current density $J_{c} \sim 10^{8} \mathrm{~A} / \mathrm{m}^{2}$, we estimate $[6,7,10]$ the drag force on the magnet to be only a small fraction $\sim 0.0001$ of the force required to enter the superconducting pipe. Thus, our results should not be much affected even if more realistic type II superconductors are used.

\section{ACKNOWLEDGMENTS}

This work is partially supported by CNPq, Brazil. We would like to thank Renato Pakter, Paulo Pureur, Osvaldo Schilling, and Fernando L. da Silveira for interesting discussions. 
[1] W. M. Saslow, Am. J. Phys. 60, 693 (1992).

[2] J. D. Jackson, Classical Electrodynamics (Wiley, New York, 1975).

[3] G. Bardotti, B. Bertotti, and L. Gianolio, J. Math. Phys. 5, 1387 (1964).

[4] J. W. Thomasson and D. M. Ginsberg, Rev. Sci. Instrum. 47, 387 (1976).

[5] J. R. Claycomb and J. H. Miller, Rev. Sci. Instrum. 70, 4562
(1999).

[6] L. C. Davis, J. Appl. Phys. 67, 2631 (1990).

[7] O. Schilling, Semicond. Sci. Technol. 17, L17 (2004).

[8] A. M. Campbell, J. Phys. C 4, 3186 (1971).

[9] Y. Levin, F. L. da Silveira, and F. B. Rizzato, Am. J. Phys. 74, 815 (2006).

[10] C. Bean, Rev. Mod. Phys. 36, 31 (1964).

[11] Y. Levin, Europhys. Lett. 76, 163 (2006). 